\title{
VALIDAÇÃO DA LIMPEZA DE PRODUTOS PARA SAÚDE NO COTIDIANO DO CENTRO DE MATERIAL E ESTERILIZAÇÃO
}

Validation of daily medical device cleaning in the sterile processing department

\author{
Validación de la limpieza de productos de salud \\ en todos los días del centro de material y esterilización
}

\author{
Rafael Queiroz de Souza ${ }^{1 *}(\mathbb{D})$, Ana Tércia Barijan² (D), Jeane Aparecida Gonzalez Bronzatti ${ }^{3}$ (D), \\ Paulo Roberto Laranjeira ${ }^{4}$ (1), Kazuko Uchikawa Graziano $0^{5}$ (D)
}

RESUMO: Objetivo: Discutir os aspectos que devem ser considerados na validação concorrente da limpeza no Centro de Materiais e Esterilização (CME). Método: Revisão narrativa da literatura científica, legislação e normatização pertinentes. Resultados: A validação da limpeza na rotina deve considerar: o design dos produtos, a definição e a exequibilidade dos procedimentos operacionais padrão, além da estrutura do CME, dimensionamento, seleção e treinamento de pessoal, registro e interpretação dos resultados obtidos pelos testes químicos na rotina. Conclusão: A validação concorrente da limpeza dos produtos para saúde no CME imprime a cultura da valorização dessa etapa do processamento entre todos os colaboradores do setor, de tal forma que a limpeza passa a ser, de fato, o núcleo central do processamento.

Palavras-chave: Enfermagem de centro cirúrgico. Enfermagem perioperatória. Segurança de produtos ao consumidor. Garantia da qualidade dos cuidados de saúde. Controle de qualidade.

ABSTRAC: Objective: To discuss the aspects that should be considered in the concurrent cleaning validation at Sterile Processing Department (SPD). Method: Narrative review of scientific literature, legislation, and pertinent normalizations. Results: The routine cleaning validation should consider the product design, definition, and feasibility of standard operating procedures (SOP); SPD structure; staff sizing; selection and training; and the recording and interpretation of results obtained by routine chemical tests. Conclusion: The concurrent cleaning validation of health products at SPD points out the value of this stage to all employees in the sector such that cleaning becomes a core function of health service product processing. Keywords: Operating Room Nursing. Perioperative Nursing. Consumer Product Safety. Quality Assurance, Health Care. Quality Control.

RESUMEN: Objetivo: Discutir los aspectos que deben considerarse en la validación concurrente de limpieza en el Centro de Materiales y Esterilización (CME). Método: revisión narrativa de la literatura científica relevante, legislación y normas. Resultados: La validación de la limpieza en la rutina debe considerar: el diseño de los productos, la definición y la viabilidad de los procedimientos operativos estándar, además de la estructura del CME, dimensionamiento, selección y capacitación del personal, registro e interpretación de los resultados obtenidos por las pruebas químicas en el rutina Conclusión: La validación concurrente de la limpieza de productos de salud en CME impresiona la cultura de valorar esta etapa de procesamiento entre todos los empleados del sector, de tal manera que la limpieza se convierta, de hecho, en el núcleo central del procesamiento.

Palabras clave: Enfermería de quirófano. Enfermería perioperatoria. Seguridad de productos para el consumidor. Garantía de la calidad de atención de salud. Control de calidad.

'Enfermeiro; pós-doutor em ciências. Pesquisador e professor de cursos de pós-graduação lato e stricto sensu; palestrante contratado de empresas de processamento de produtos para saúde e segurança do paciente - São Paulo (SP), Brasil.

¿Enfermeira; mestre em Ciências. Especialista de assuntos científicos e educacionais da Divisão de Soluções Médicas da 3M do Brasil - Sumaré (SP). Brasil

${ }^{3}$ Enfermeira; doutora em ciências pela Escola de Enfermagem da Universidade de São Paulo (USP). Pesquisadora e professora em cursos de pós-graduação lato sensu no Brasil e stricto sensu no Peru pela Universidad Alcalá. Consultora Técnica em Saúde; palestrante contratada por empresas para abordar assuntos nas áreas de assistência cirúrgica e processamento de produtos para saúde - São Paulo (SP), Brasil. «Engenheiro elétrico; doutor em ciências. Gerente da Garantia de Esterilização da Cardinal Health. Palestrante da 3M; membro da Association for the Advancement of Medical Instrumentation - Estados Unidos. ${ }^{5}$ Enfermeira. Professora titular sênior do Departamento de Enfermagem Médico-Cirúrgica da Escola de Enfermagem da Universidade de São Paulo; coordenadora pedagógica do curso MBA/CME, da Faculdade CEAT - São Paulo (SP), Brasil.

*Autor correspondente: rafaelqsouza@hotmail.com

Recebido: 12/10/2019 - Aprovado: 19/01/2020

DOI: $10.5327 / Z 1414-4425202000010009$ 


\section{INTRODUÇÃo}

A validação dos procedimentos operacionais padrão(POP) tem sido tema recorrente nas discussões envolvendo a segurança no processamento de produtos para saúde (PPS). A crescente preocupação com o tema justifica-se, principalmente, pela complexidade dos produtos destinados aos procedimentos cada vez menos invasivos, que impôs desafios crescentes ao CME, como as pinças para cirurgia robótica e os endoscópios digestivos com canais inacessíveis à escovação.

No contexto apresentado, a limpeza destaca-se por ser o procedimento fundamental que torna os produtos seguros para manuseio e os prepara para desinfecção ou esterilização ${ }^{1}$. Nos Estados Unidos da América (EUA), a Food and Drug Administration (FDA) publicou um guia próprio com orientações para a validação das instruções de uso (IDU) para o processamento de produtos em serviços de saúde 2 . No Brasil, houve iniciativas semelhantes, como a tradução da Norma ISO 17.664", que estabeleceu as informações sobre o processamento que devem ser fornecidas pelo fabricante aos usuários, assim como a Resolução da Diretoria Colegiada (RDC) da Agência Nacional de Vigilância Sanitária (Anvisa) RDC 15/2012 ${ }^{1}$, exigindo que cada etapa do processamento siga um POP elaborado com base no referencial científico atualizado e normatização pertinente.

Para que os POP sejam validados, invariavelmente, são empregados métodos laboratoriais sofisticados para detecção de resíduos orgânicos, com alta sensibilidade e procedimentos normatizados, como aqueles publicados pela International Organization for Standardization (ISO), Association for the Advancement of Medical Instrumentation (AAMI), FDA e farmacopeias. Embora necessários nas validações prospectivas, esses métodos muitas vezes não são passíveis de utilização na rotina e, frequentemente, os profissionais questionam: como assegurar que os resultados validados pelo fabricante estão sendo alcançados na prática? Adicionalmente, pela falta de direcionamento claro por parte dos órgãos reguladores e padronização do processo de validação, os fabricantes podem fornecer IDU inconsistentes com as boas práticas, sem comprovação da validação, comprovadas sob condições que não simulam um CME e, algumas vezes, impossíveis de serem seguidas, causando desconfiança sobre sua aplicabilidade.

Para responder a essa dúvida, inicialmente, deve ser retomado o conceito de validação, que significa produzir uma evidência objetiva e passível de confirmação dos requisitos específicos para que uma determinada finalidade possa ser consistentemente cumprida ${ }^{4}$. Nos estudos que envolvem a validação dos processos de limpeza, a obtenção de resultados consistentes é possível por meio da elaboração e do cumprimento de um $P O P$, que visa à padronização e à redução na variabilidade dos resultados.

Os resultados consistentes em cenário laboratorial são facilitados pelo controle de variáveis, porém, na área da limpeza do $\mathrm{CME}$, muitos fatores comprometedores do POP podem estar presentes, sejam estruturais (área física subdimensionada), tecnológicos (equipamentos obsoletos e passíveis de falha), materiais (consumíveis, como detergentes) e humanos (quadro de pessoal e variação nas características individuais como força física, habilidade, familiaridade com o POP), entre outros ${ }^{2}$. Dessa forma, além do POP, outro procedimento necessário para assegurar a qualidade é o monitoramento, que pode ser realizado por meio da inspeção com lentes de aumento e testes químicos para uso em $\mathrm{CME}^{1}$, fáceis de incorporar à rotina.

Com base nas informações apresentadas, este estudo visa discutir os aspectos que devem ser considerados na validação concorrente da limpeza no CME. Ressalta-se que não se pretende substituir métodos descritos na normatização pertinente, que devem ser utilizados pelos fabricantes na validação prospectiva de IDU, porém tem como finalidade fundamentar os profissionais na validação de POP de limpeza no cotidiano do CME.

\section{OBJETIVO}

Discutir os aspectos que devem ser considerados na validação concorrente da limpeza de produtos para saúde no CME.

\section{MÉTODO}

Trata-se de uma revisão narrativa e crítica da literatura, incluindo documentos legais e normativos, nacionais e internacionais, que embasam procedimentos de validação e suas interfaces com a limpeza de PPS no cotidiano do CME.

\section{RESULTADOS E DISCUSSÃO \\ Característica dos produtos passíveis de processamento}

Os produtos passíveis de processamento permitem repetidos processos de limpeza, preparo e desinfecção ou esterilização ${ }^{1}$, entretanto diversas características podem influenciar a 
limpeza como: ser desmontável para favorecer a limpeza de áreas de difícil acesso; ser transparente para permitir a visualização da sujidade; ter estrutura sólida para evitar o acúmulo de sujidade; ter estrutura interna que permite a entrada e a saída de água, facilitando a remoção de sujidade pela ação mecânica da água ${ }^{5}$; além da qualidade do acabamento das superfícies internas.

Na prática, nota-se que nem sempre o design dos produtos favorece a limpeza e a dificuldade de remoção da sujidade, que é aumentada pelo tipo de matéria orgânica como sangue, ossos e gordura. Adicionalmente, a literatura científica tem demonstrado que produtos processados rotineiramente pelo CME, como fresas intramedulares flexíveis, não são passíveis de limpeza em razão da sua conformação ${ }^{6}$. Portanto, é necessária uma análise criteriosa da possibilidade de limpeza de cada produto, considerando não somente a capacidade técnica como o design acessível.

\section{Estrutura para implantação dos POP}

A validação concorrente dos POP de limpeza é necessária em razão da variedade de insumos e equipamentos nos CME. Embora as IDU contenham todas as informações necessárias para limpeza ${ }^{3}$, na prática nota-se que o mercado oferece diferentes equipamentos, soluções e artefatos para higienização como escovas, esponjas, dispositivos pull thru TM, entre outros. Há serviços que predominam processos manuais, em outros os processos automatizados, porém os $\mathrm{CME}$ só podem processar produtos compatíveis com sua capacidade técnica operacional e classificação de infraestrutura ${ }^{1}$.

Para que a constância nos resultados da limpeza seja assegurada, os equipamentos utilizados devem ser submetidos às qualificações de instalação, de operação e de desempenho, com periodicidade mínima anual ${ }^{1}$. Os equipamentos devem ser qualificados dentro do intervalo habitualmente estabelecido de 12 meses ou toda vez que passarem por intervenções de manutenção, mudança de local e suspeita de falhas ${ }^{1,7}$. Esses procedimentos devem estar de acordo com a avaliação de mudança, que consiste em um protocolo de avaliação de mudanças dos pontos críticos de funcionamento do equipamento e da rotina, determinando qual qualificação deverá ser refeita para garantir que o equipamento continue qualificado e o processo validado ${ }^{7}$.

Esses procedimentos têm como objetivo garantir que os equipamentos foram entregues e instalados de acordo com as suas especificações, que operam dentro dos parâmetros originais de fabricação e que apresentam desempenho consistente com parâmetros idênticos, utilizando-se a carga de maior desafio definida pelo $\mathrm{CME}^{1}$. De acordo com os fabricantes, os equipamentos podem exigir procedimentos de controle a cada uso, diários, trimestrais, anuais ou de acordo com o controle de mudanças estabelecido em parceria com o serviço de engenharia clínica ${ }^{8}$. Entretanto, há equipamentos, como as lavadoras ultrassônicas e os sistemas de limpeza por vapor fluente, que ainda não possuem normas técnicas construtivas e de qualificação, sendo recomendado que as IDU dos respectivos fabricantes sejam seguidas. Em geral, para a conservação e operação dos equipamentos de limpeza, os fabricantes também recomendam os critérios estabelecidos na norma ABNT NBR ISO 17665-29, em relação à qualidade da água que abastece os equipamentos.

Quanto às soluções de limpeza, atenção especial deve ser dada às IDU, incluindo as informações sobre diluição, qualidade da água para preparo, tempo de imersão, faixa de temperatura e $\mathrm{pH}$. Adicionalmente, o CME deverá dispor de escovas compatíveis com comprimento, diâmetro e IDU do produto, cerdas macias, para não danificar as superfícies internas, e com comprimento das cerdas suficiente para promover o atrito na superfície ${ }^{10}$.

Por fim, a validação dos procedimentos de limpeza está condicionada não só à estrutura disponível no CME, como aos equipamentos e produtos consumíveis, mas também à evidência documentada de que estão em condições de uso, por meio de verificação periódica.

\section{Definição dos POP}

Em linhas gerais, o POP é um documento oficial que descreve cada passo crítico e sequencial a ser dado pelo operador, a fim de garantir o resultado esperado de uma tarefa ${ }^{11}$, devendo ser amplamente divulgado e elaborado com base na literatura científica e na normatização relacionada ${ }^{1}$. Uma vez que o POP é uma sequência padronizada para realização de um determinado procedimento, está intimamente ligado ao treinamento dos profissionais que atuam na área de limpeza.

A IDU dos PPS inclui todas ou algumas atividades de preparação no ponto de uso; preparo; limpeza; desinfecção; secagem; inspeção; manutenção; ensaios; embalagem; esterilização e armazenamento, sendo, portanto a base para a definição de um POP para processamento de produtos no $\mathrm{CME}^{3}$. Quaisquer atividades relacionadas ao processamento devem estar de acordo com a regulação nacional pertinente e com recomendações baseadas em evidências de organizações de renome, sejam nacionais ou internacionais. 
Entretanto, a avaliação das IDU fornecidas pelo fabricante requer análise minuciosa, principalmente no Brasil, que podem ser fornecidas sem validação. A literatura tem reportado recomendações inconsistentes, erros conceituais graves e procedimentos equivocados em IDU ${ }^{12}$. Somente o fabricante dos PPS pode fornecer a IDU. Caso seja traduzida pelo distribuidor, essa deve ser oficial (juramentada) ou o usuário poderá solicitar a original para verificar o conteúdo. Cuidados adicionais devem ser tomados com relação aos erros de tradução.

Quaisquer procedimentos devem ter seus passos claramente descritos e tecnicamente possíveis de execução pelo pessoal do CME. Os POP com tempo de execução excessivamente longo e com muitas etapas manuais de limpeza podem levar à normalização dos desvios e inconsistência, constituindo um grande desafio para o CME, além de comprometer seriamente a efetividade da limpeza, especialmente em serviços com pouca infraestrutura e/ou alta demanda ${ }^{6}$.

O tempo total de execução de um determinado POP pode também ser comprometido pela logística para recebimento de produtos consignados pelo CME. A entrega desses produtos fora do prazo definido pelo CME é uma realidade e pode constituir um potencial fator para o não cumprimento dos POP.

\section{Dimensionamento e treinamento de pessoal}

No Brasil, historicamente, as atividades operacionais desenvolvidas no CME, em grande parte, foram realizadas por técnicos e auxiliares de enfermagem, enquanto a gestão técnica e administrativa era realizada pelos enfermeiros. Apesar do papel fundamental que o CME desempenha na qualidade do processo assistencial, verifica-se que frequentemente esse setor conta com um quadro de pessoal insuficiente ou sem qualificação adequada ${ }^{13}$.

Todas as etapas do processamento de PPS devem ser realizadas por profissionais para os quais essas atividades estejam regulamentadas pelos seus conselhos de classe ${ }^{1}$. Diante dessa situação, o Conselho Federal de Enfermagem (COFEN) publicou a Resolução n ${ }^{\circ} 424$ / 2012, a qual normatiza as atribuições dos profissionais de enfermagem no CME e em empresas processadoras de PPS ${ }^{14}$.

Recentemente, considerando a necessidade de se fazer revisão e atualização de parâmetros que subsidiavam o planejamento, o controle, a regulação e a avaliação das atividades assistenciais de enfermagem, o COFEN estabeleceu a Resolução $n^{\circ} 0543 / 2017^{15}$. Atualmente, o dimensionamento de pessoal precisa considerar questões como: missão; visão; porte; política de pessoal; recursos materiais e financeiros; estrutura organizacional e física; tipos de serviços e/ou programas; tecnologia e complexidade dos serviços e/ ou programas; atribuições e competências específicas dos integrantes dos diferentes serviços ${ }^{15}$.

Devem-se considerar, também, os aspectos técnico-científicos e administrativos, como a dinâmica de funcionamento das unidades nos diferentes turnos, o modelo gerencial, os métodos de trabalho, a jornada de trabalho, a carga horária semanal, os padrões de desempenho dos profissionais, o índice de segurança técnica (IST), a proporção de profissionais de enfermagem de nível superior e de nível médio e os indicadores de qualidade ${ }^{15}$.

Mesmo adequando o quantitativo de profissionais no CME, é preciso estabelecer a competência mínima que o indivíduo deve ter para exercer as atividades desse setor. Por se tratar de um serviço no qual as tarefas envolvem procedimentos muito específicos, deve-se valorizar, além da competência técnica, o aprimoramento e o desenvolvimento por meio de uma educação permanente.

Os profissionais que atuam no CME devem receber a orientações iniciais abordando todas as tarefas realizadas na área de PPS, mesmo as relacionadas às políticas e aos procedimentos de prevenção e controle de infecção, segurança, vestuário, higiene pessoal, legislações e regulamentações estaduais e federais ${ }^{16}$.

É recomendado que um programa de educação continuada em intervalos regulares seja utilizado no CME, com o objetivo de revisar e atualizar o conhecimento e as habilidades, mantendo assim competência profissional, além de treinamentos extraordinários, sempre que houver a inserção de novos produtos, equipamentos e procedimentos ${ }^{16}$. $\mathrm{O}$ trabalho associado à orientação, educação e treinamento fornece aos trabalhadores informações essenciais para executar com responsabilidade as atividades a eles designadas, diminuir os riscos de erros operacionais e garantir a familiarização com as técnicas utilizadas ${ }^{16}$.

Os profissionais que atuam no CME e nas empresas processadoras devem receber capacitação específica e periódica nos seguintes temas: classificação de PPS; conceitos básicos de microbiologia; transporte dos produtos contaminados; processo de limpeza; desinfecção; preparo; inspeção; acondicionamento; embalagens; esterilização; funcionamento dos equipamentos; monitoramento de processos por indicadores químicos, biológicos e físicos; rastreabilidade; armazenamento e distribuição dos PPS e manutenção da esterilidade do produto ${ }^{1}$. Acrescenta-se também o treinamento específico para uso dos equipamentos de proteção individual (EPI) adequados às atividades desenvolvidas no $\mathrm{CME}^{1}$. 
Embora os gestores de CME reconheçam a necessidade e a importância do treinamento permanente para os colaboradores, o ritmo de trabalho imposto no setor raramente permite que os profissionais se ausentem dos seus postos de trabalho para receberem os treinamentos mínimos exigidos pela Anvisa ou sobre novos procedimentos. Para validar processos de limpeza, é imprescindível ter, além de todas as etapas padronizadas, profissionais comprometidos e preparados para executar os POP.

\section{Testes químicos}

Um relatório de 234 eventos relacionados aos PPS cirúrgicos associou $34 \%$ das causas à limpeza inadequada e destacou que a presença de sujidade foi detectada com o paciente em sala operatória ${ }^{17}$. Esses dados mostraram que houve falha na inspeção e no monitoramento, que são importantes ferramentas para a garantia da qualidade no $\mathrm{CME}^{16}$.

O monitoramento da limpeza avalia a presença de resíduos orgânicos e inorgânicos no instrumental como sangue; biofilme; gorduras; fragmentos de tecido; secreções corporais como fezes; secreções respiratórias; microrganismos; cimentos ósseos; visco elástico; sais; entre outros ${ }^{16}$. Esse procedimento deve ser realizado por meio da inspeção visual, com o auxílio de lentes intensificadoras de imagem, complementada, quando indicado, por testes químicos disponíveis no mercado $^{1}$. Atualmente, alguns CME já possuem tecnologias de amplificação de imagem com maior resolução, que são um excelente recurso para o monitoramento, bem como para a inspeção de funcionalidade, detectando fadiga e início de corrosão em pequenas estruturas.

Alguns marcadores podem auxiliar no monitoramento da limpeza, como: proteína; hemoglobina; carga microbiana e adenosina trifosfato (ATP $)^{16}$. Outros testes ainda não são possíveis na rotina: carboidratos; endotoxinas; gorduras e sódio; porém, podem ser realizados em laboratórios. Os testes de rotina podem ser empregados para avaliação do desempenho dos funcionários, efetividade dos POP de limpeza e funcionalidade de equipamentos ${ }^{16}$.

Os testes químicos fornecem informações quantitativas, por exemplo, unidades relativas de luz (RLU) para detecção de ATP. Os valores de corte podem ser determinados com base em referencial científico ou, quando não disponíveis, pela análise de tendência da série histórica, sendo fundamental que os valores obtidos sejam armazenados. Outro exemplo são testes semi-quantitativos, que mudam de cor quando um determinado tipo de sujidade se encontra acima de valores de referência pré-estabelecidos. Antes de determinar a escolha do teste, deve-se levar em consideração a IDU, bem como as indicações e limitações de cada tecnologia.

A verificação da limpeza deve incluir resultados da inspeção e testes químicos, incluindo as partes internas (lúmens) e as externas (superfícies), testes de eficácia dos equipamentos empregados no processo (testes específicos oferecidos por diversos fabricantes) e monitoramento de parâmetros críticos de processo, como temperatura utilizada (em geral, obtida nos impressos e registros realizados pelo próprio equipamento $)^{16}$.

Os responsáveis técnicos e gestores do CME devem estabelecer níveis adequados de qualidade para os produtos e serviços que produzem e garantir que esses níveis sejam consistentemente mantidos ${ }^{18}$. Portanto, mais importante que a escolha de um ou mais testes é a gestão das informações que eles fornecem para o controle da qualidade do CME.

O conceito de qualidade diz respeito ao grau de excelência de um produto ou serviço e uma das formas de avaliar, isto é, por meio de indicadores. Os resultados dos indicadores podem indicar inconsistências no processo, as quais poderão ser solucionadas no momento em que se faz análise de causa, identifica-se o problema e propõe-se as mudanças para solucioná-los ${ }^{16,18}$. Portanto, a implantação de quaisquer testes químicos deve ser acompanhada das ferramentas de qualidade, a exemplificar: diagrama de Pareto, ciclo PDCA (plan, do, check, act; em português: planejar, fazer, verificar e agir), folha de verificação, diagrama de dispersão, causa e efeito.

$\mathrm{Na}$ rotina do $\mathrm{CME}$, observa-se que há registro dos resultados dos testes, de acordo com periodicidade definida, porém, muitas vezes encontram-se resultados não satisfatórios e não existe nenhum plano de ação, nem tão pouco um plano de contingência. Além disso, muitas vezes, os profissionais realizam adaptações indevidas das tecnologias de monitoramento como um produto que foi desenvolvido para monitoramento de lavadoras termodesinfectadoras, sendo utilizado em lavadoras ultrassônicas.

\section{Amostragem e controles comparativos na validação concorrente}

Na validação concorrente da limpeza, tem que se estabelecer critérios para selecionar os PPS que serão examinados pelo método visual magnificado ou por testes químicos comercialmente disponíveis ${ }^{1}$, posto que é impossível e desnecessário legitimar a limpeza de todos os PPS processados no dia a dia por um CME. Este procedimento, caracterizado como amostragem $^{19}$, deverá seguir critérios deliberadamente definidos, por exemplo, pela complexidade da conformação na 
perspectiva de que, "quanto mais complexo o PPS, mais difícil a remoção da sujidade retida". Nesse sentido, os PPS que possuem lúmens, sem dúvida, devem todos passar por controles, não só da avaliação da limpeza dos espaços internos, mas se os lúmens estão pérvios passando hastes rígidas em busca de, por exemplo, resíduos sólidos como restos de osso em instrumentos ortopédicos canulados ${ }^{6}$. Outro exemplo é a avaliação da presença de resíduos da solução viscoelástica solidificada em cânulas de hidrodissecção. A taxa de materiais encontrados sujos, dividida pelo número total de unidades examinadas, constituirá o indicador de qualidade da limpeza, sinalizando problemas que devem ser solucionados. Estes podem ser relacionados aos recursos humanos executantes da limpeza, produtos e insumos utilizados, desempenho dos equipamentos e sobrecargas de trabalho no setor de limpeza. Outros critérios racionais sugestivos para amostragem podem ser: monitorar a qualidade de limpeza dos colaboradores iniciantes na atividade; monitorar o desempenho de lavadoras recém-adquiridas; obsoletas ou após manutenções; monitorar a limpeza de PPS complexos; entre outros.

Ressalta-se que não deve ser subestimada a validação concorrente da limpeza dos PPS limpos pelos métodos automatizados. Os equipamentos podem ter desempenhos diferenciados conforme seu histórico e sua procedência, manutenções preventivas programadas, tempo de uso e, mesmo aqueles considerados "excelentes" na qualificação de desempenho, falham inesperadamente num ciclo devido, por exemplo, a "área de sombra", no qual a ação mecânica do jato sob pressão ou ultrassom é menor ou até ausente. Isso posto, a AAMI recomenda o teste diário dos equipamentos ${ }^{16}$.

Além desse caso, o problema pode, também, estar relacionado à falha humana no carregamento das máquinas, aspecto esse de controle mais difícil, porém não menos importante.

\section{CONSIDERAÇÕES FINAIS}

Por meio deste estudo narrativo e crítico, demonstrou-se que a validação concorrente está além do simples cumprimento dos POP, pois contempla aspectos relacionados ao design dos produtos, exequibilidade do $\mathrm{POP}$, estrutura do $\mathrm{CME}$, dimensionamento e treinamento de pessoal, seleção, documentação e interpretação dos resultados obtidos pelos testes químicos.

A prática da validação concorrente da limpeza dos PPS no CME imprime a cultura da valorização dessa etapa do processamento entre todos os profissionais que atuam nessa área, de tal forma que a limpeza passa a ser, de fato, o núcleo central do processamento.

\section{REFERÊNCIAS}

1. Brasil. Ministério da Saúde. Resolução da diretoria colegiada no 15 , de 15 de março de 2012. Dispõe sobre requisitos de boas práticas para o processamento de produtos para saúde e dá outras providências. Brasília: Ministério da Saúde; 2012.

2. U.S. Food and Drug Administration. Reprocessing medical devices in health care settings: validation methods and labeling. Guidance for industry and food and drug administration staff [Internet]. 2015 [acessado em 30 maio 2019]. Disponível em: https://www.fda. gov/downloads/medicaldevices/deviceregulationandguidance/ guidancedocuments/ucm253010.pdf

3. Associação Brasileira de Normas Técnicas (ABNT). NBR ISO 17664:2015: Esterilização de produtos para saúde - Informação a ser fornecida pelo fabricante para o processamento de produto para saúde resterilizável. Rio de Janeiro: ABNT; 2015.

4. U.S. Food and Drug Administration. Code of Federal Regulations Title 21 Part 820 . Quality system regulation [Internet]. [acessado em 30 maio 2019]. Disponível em: https://www.accessdata.fda.gov/ scripts/cdrh/cfdocs/cfcfr/CFRSearch.cfm?CFRPart=820
5. Graziano KU, Bálsamo AC, Lopes CLBC, Zotelli MFM, Couto AT, Paschoal MLH. Critérios para avaliação das dificuldades na limpeza de artigos de uso único. Rev Latino-Am Enferm. 2006;14(1):70-6. http://dx.doi.org/10.1590/S0104-11692006000100010

6. Souza R, Bronzatti J, Laranjeira P, Mimica L, Silva C, Cruz A, et al. Avaliação da segurança do processamento de fresas intramedulares flexíveis para cirurgia ortopédica. Rev SOBECC. 2017;22(1):17-22. https://doi.org/10.5327/ Z1414-4425201700010004

7. Associação Brasileira de Normas Técnicas (ABNT). NBR ISO 17665-1, Esterilização de produtos para saúde - Vapor - Parte 1: Requisitos para o desenvolvimento, validação e controle de rotina nos processos de esterilização de produtos para saúde. Rio de Janeiro: ABNT; 2010.

8. Laranjeira P, Bronzatti J, Souza R, Graziano K. Fundamentos para uso seguro das lavadoras termodesinfetadoras com ênfase na liberação para uso após intervenção técnica. Rev SOBECC. 2016;21(3):178-84. https://doi.org/10.5327/Z1414-4425201600030009 
9. Associação Brasileira de Normas Técnicas (ABNT). NBR ISO 176652, Esterilização de produtos para saúde - Vapor - Parte 2: Guia de aplicação da ABNT NBR ISO 17665-1. Rio de Janeiro: ABNT; 2013.

10. Bajolet O, Ciocan D, Vallet C, De Champs C, Vernet-Garnier V, Guillard T. et al. Gastroscopy-associated transmission of extended-spectrum beta-lactamase producing Pseudomonas aeruginosa. J Hosp Infect. 2013;83(4):341-3. https://doi.org/10.1016/j.jhin.2012.10.016

11. Nogueira LCL. Gerenciando pela qualidade total na saúde. $3^{\mathrm{a}}$ ed. Nova Lima: INDG Tecnologia e Serviços LTDA., 2008.

12. Bruna C, Souza R, AlmeidaA, SuzukiK, Turrini R, Graziano K. Processamento de cabos de laringoscópio: revisão integrativa. RevSOBECC. 2016;21(1):3745. https://doi.org/10.5327/Z1414-4425201600010006

13. Costa JA, Fugulin FMT. Atividades de enfermagem em centro de material e esterilização: contribuição para o dimensionamento de pessoal. Acta Paul Enferm. 2011;24(2):249-56. https://doi.org/10.1590/ S0103-21002011000200015

14. Conselho Federal de Enfermagem (COFEN). Resolução COFEN ${ }^{\circ}$ 424/2012. Normatiza as atribuições dos profissionais de enfermagem em Centro de Material e Esterilização (CME) e em empresas processadoras de produtos para saúde [Internet]. Brasília: COFEN; 2012 [acessado em 17 jan. 2020]. Disponível em: www.cofen.gov.br/resoluo-cofen-n-4242012_8990.html
15. Conselho Federal de Enfermagem (COFEN). Resolução COFEN $n^{\circ}$ 0543/2017. Atualiza e estabelece parâmetros para o dimensionamento do quadro de profissionais de enfermagem nos serviços/locais em que são realizadas atividades de enfermagem [Internet]. Brasília: COFEN; 2017 [acessado em 10 out. 2018]. Disponivel em: http://www.cofen.gov.br/resolucaocofen-5432017_51440.html

16. American National Standard/Advancing Safety in Healthcare Technology -ANSI/AAMI ST79:2017. Comprehensive guide to steam sterilization and sterility assurance in health care facilities. Arlington: American National Standard; 2017.

17. Event Reporting \& Analysis - Alerts. If it's not clean, it's not sterile: reprocessing contaminated instruments [Internet]. ECRI Institute; 2017 [acessado em 10 out. 2018]. Disponível em: https://www.ecri.org/components/PSOCore/Pages/e-lert041117. aspx?PF=1? source=print

18. International Association of Healthcare Central Service Material Management (IAHCSMM). Quality Assurance. Chicago: IAHCSMM; 2016.

19. Silva NN. Amostragem probabilística: um curso introdutório. São Paulo: Edusp; 2001. 